\title{
Opportunistic Spectrum Sharing with Multiple Cochannel Primary Transmitters
}

\author{
Ahmed O. Nasif, Student Member, IEEE, and Brian L. Mark, Senior Member, IEEE
}

\begin{abstract}
We present a distributed, collaborative algorithm to enable opportunistic spectrum access for cognitive radios in the presence of multiple cochannel transmitters. A spectrum hole detection and estimation technique based on received signal strength observations is developed, which allows the coexistence of both licensed and unlicensed transmitters. We address the issues of how to perform collaborative spectrum sensing in the presence of multiple cochannel transmitters and how to determine the maximum transmit power that can be used for a given frequency channel by a cognitive radio while avoiding harmful interference to the licensed network. Simulation results are provided to validate the feasibility and performance of the proposed scheme.
\end{abstract}

Index Terms-Spectrum sharing, cognitive radio, radio resource management, geolocation.

\section{INTRODUCTION}

$\mathbf{R}$ ECENTLY there has been much interest in cognitive radios (CRs) and their application to opportunistic spectrum access (OSA) to maximize the utilization of licensed spectrum [2], [3]. CRs equipped with features like frequency agility, high receiver sensitivity and location-awareness, are seen as a promising technology to allow the non-disruptive co-existence of unlicensed (secondary) users alongside the licensed (primary) users. Most of the proposed OSA schemes in the literature can be categorized into coordinated (the so-called property-rights model) and uncoordinated (the socalled commons model) frameworks [3]. In the coordinated approach, the primary and secondary nodes can exchange information and cooperatively increase spectrum utilization [4]. In uncoordinated OSA, which is the model of interest for this paper, the primary is oblivious to the existence of secondary nodes, and the secondary system senses the activity of the primary system to opportunistically use the same spectrum, provided that no harmful interference is caused to the primary. In other words, the secondary system tries to fill the "spectrum holes," which may represent opportunities in time or space or both.

OSA in the time domain has been studied extensively using the tools of information theory [5], game theory [6], queueing theory [7], and partially observable Markov chains

Manuscript received March 4, 2009; revised July 16, 2009; accepted August 15,2009 . The editor coordinating the review of this paper and approving it for publication was T. Hou.

The authors are with the Dept. of Electrical and Computer Engineering, George Mason University, Fairfax, VA 22030 (e-mail: \{anasif, bmark\}@gmu.edu).

This work was supported in part by the U.S. National Science Foundation under Grant CNS-0520151 and ECS-0426925. An early version of this work was presented in part at IEEE Globecom'08, Dec. 2008 [1].

Digital Object Identifier 10.1109/TWC.2009.090316
[8]. Channel sensing mechanisms, detector design and effect of collaboration among secondary nodes have received considerable attention [9]-[11]. On the other hand, the use of location information and localization to exploit spatial spectrum holes have received relatively limited attention.

Localization for cognitive radio networks poses unique challenges, such as lack of coordination with the primary system and the need for robustness against a wide range of operating conditions. The enhancement of cognitive capabilities with location information, which can be utilized to perform dynamic spectrum management, network planning and handover is discussed in [12]. To ensure the operation of CRs under different environments, a cognitive positioning system (CPS) based on time-of-arrival (TOA) is proposed in [13]. Localization using signal strength (SS) measurements of a primary transmitter with unknown transmit power based on a constrained least squares approach is considered in [14]. Localization involving multiple primary transmitters is studied in [15], [16], where it is assumed that the number of transmitters and their transmit powers are known a priori. An experimental study, employing a triangulation-based heuristic approach for multiple transmitter localization using synchronized sensing is presented in [17]. Range-free localization of the primary is proposed in [18], while the use of spatial statistics to characterize CR networks is suggested in [19]. Many of the existing works on spatial spectrum sensing assume a single primary transmitter scenario and knowledge of the transmitter's location and transmit power [20]-[22].

A simple Listen-Before-Talk (LBT) algorithm is analyzed in [23], where the spectrum hole is characterized in terms of the maximum interference-free transmit power (MIFTP). The MIFTP is defined as the maximum power level at which a secondary node can transmit without causing harmful interference to the primary users. In [24], we proposed an approach to collaborative spectrum hole detection and estimation based on signal strength observations obtained by a group of secondary nodes with respect to a single primary transmitter.

In this paper, we present a collaborative sensing scheme designed to accommodate the presence of multiple cochannel transmitters, which may arise, for example, in cellular systems. Given a set of measurements, the first task is to identify the total number of cochannel primary transmitters and perform measurement clustering, so that the clustered secondary nodes can form groups and estimate the parameters of the primary transmitter in their vicinity, ignoring the effect of cochannel interference temporarily. Subsequently, the clustered groups can share the estimated parameters of the transmitters located in their vicinity with other groups to improve the initial 
estimates. This results in a distributed and iterative method to mitigate the effect of cochannel interference in spatial sensing.

We show that spatial spectrum holes can be identified accurately provided that locally sensed information about cochannel transmitters is shared among the secondary nodes. In particular, we propose the maintenance of a distributed database, called the T-map, containing cochannel transmitter information including location, power, error estimates, and other information. Once all the parameters of the transmitters are estimated, a method using the T-map is proposed to determine the approximate MIFTP that can be allocated to a particular secondary node without causing harmful interference to the existing cochannel primary nodes.

The remainder of the paper is organized as follows. Section II describes the OSA model in detail. Section III presents a collaborative and distributed localization-based spatial spectrum sensing scheme to mitigate cochannel interference. An approximation for the MIFTP in the presence of multiple transmitters is developed in Section IV. Section V, presents some numerical results to validate the feasibility and performance of our proposed approach. Finally, the paper is concluded in Section VI.

\section{Collaborative Spectrum Sharing Model}

Consider a group of CRs deployed in the coverage area of a licensed network consisting of multiple primary transmitters operating on a given channel $\nu \in \mathcal{C}$, where $\mathcal{C}$ denotes the set of channels under consideration. We propose a collaborative OSA scheme that identifies the spatial regions where the CRs can reuse the channel $\nu$, without causing harmful interference to the primary receivers and to each other. In the literature, this is referred to as spectrum hole discovery. No direct communication between the primary and CR nodes is possible, but CRs can communicate with each other for robust spectrum sensing. Without loss of generality, we assume the existence of a common control channel that can be used by the CRs to exchange control information.

\section{A. SS-based observation model}

We assume that all transmissions are omnidirectional and the propagation model is homogeneous, with lognormal shadowing. The received signal strength (SS) at node $i$ due to node $j$ is denoted by

$$
R_{i j}=s_{j}-g\left(d_{i j}\right)+W_{i j}[\mathrm{dBm}],
$$

where $s_{j}$ is the transmit power of node $j, g\left(d_{i j}\right)$ is the path loss between two nodes separated by $d_{i j}$, and $W_{i j} \sim \mathcal{N}\left(0, \sigma_{W}^{2}\right)$, i.e., $W_{i j}$ is normally distributed with zero mean and variance $\sigma_{W}^{2}$. Assume that $g(d)$ is continuous, monotonically increasing and invertible. In general, $g(\cdot)$ is also a function of the path loss factor, antenna heights, antenna polarization, carrier frequency, terrain details etc., but for simplicity we assume that these other parameters can be estimated separately. Since multipath fast fading occurs on a much smaller time scale than shadowing, it is fair to assume that the fast fading can be practically eliminated by employing averaging (see [18],
[25]). The net SS received at node $i$ due to a set of cochannel transmitters $\mathcal{J}$ in $\mathrm{dBm}$ is given by

$$
R_{i}=10 \log _{10}\left(\sum_{j \in \mathcal{J}} 10^{\frac{R_{i j}}{10}}\right) .
$$

\section{B. Definition of MIFTP}

Denote the set of cochannel primary transmitters and the set of secondary nodes by $\mathcal{P}$ and $\mathcal{A}$, respectively. Each node $a \in$ $\mathcal{P} \cup \mathcal{A}$ has an associated location $\left(x_{a}, y_{a}\right)$ and transmit power $s_{a}$. The primary receivers are referred to as victim nodes, since they can potentially be disrupted by secondary transmissions. The coverage distance of primary transmitter $p \in \mathcal{P}$ is given by

$$
d_{\mathrm{cov}}(p)=g^{-1}\left(s_{p}-r_{\min }+\sigma_{W} Q^{-1}\left(1-\varepsilon_{\mathrm{cov}}\right)\right),
$$

where $s_{p}$ is the transmit power of $p, \varepsilon_{\mathrm{cov}}$ is a predefined upper limit on the outage probability of an intended receiver located inside the coverage area of $p, r_{\min }$ is the detection threshold of primary receivers (i.e., victims), $g^{-1}(\cdot)$ denotes the inverse of $g(\cdot)$, and $Q(x) \triangleq \frac{1}{\sqrt{2 \pi}} \int_{x}^{\infty} e^{-\frac{t^{2}}{2}} d t$ denotes the standard $Q$-function (cf. [24]).

We define the coverage region of $p \in \mathcal{P}$ as the closed ball or disk centered at $p$ with radius $d_{\text {cov }}(p)$, denoted by $\bar{B}_{\text {cov }}(p)$. The coverage region corresponds to the geographical area in which the received signal from $p$ is sufficiently strong to satisfy a certain quality-of-service requirement. Primary nodes residing within the coverage region are potential victim nodes, since they may be receiving transmissions from node $p$ and may experience interference from cochannel secondary transmitters. Nodes outside the coverage region will be oblivious to interference caused by secondary transmissions. Similarly, all secondary nodes detecting the signal of a particular primary transmitter $p$, must be located within the detection radius $d_{\text {det }}(p)$, defined as

$$
d_{\operatorname{det}}(p)=g^{-1}\left(s_{p}-r_{a}+\sigma_{W} Q^{-1}\left(1-\varepsilon_{\mathrm{cov}}\right)\right),
$$

where $r_{a}$ is the detection threshold of the secondary nodes.

Consider a set of existing cochannel secondary transmitters $\mathcal{A}_{T} \subset \mathcal{A}$ and a secondary node $b \in \mathcal{A} \backslash \mathcal{A}_{T}$ that is considering to reuse the same channel. Define $\mathcal{A}_{0} \triangleq \mathcal{A}_{T} \cup\{b\}$. Denote by $I_{v}$, the aggregate interference power received at a victim node $v$ due to the transmissions of nodes in $\mathcal{A}_{0}$. We ignore the effect of interference caused by cochannel primary transmitters. Typically, this would be taken into account in the design of the primary network. If this is not the case, we can simply treat the primary cochannel transmitters as secondary transmitters for the purpose of interference analysis. The interference probability with respect to $v$ is defined as the probability that $I_{v}$ exceeds a predefined threshold $i_{\max }$ :

$$
P_{\text {int }}\left(\mathcal{A}_{0}, v\right) \triangleq \operatorname{Pr}\left\{I_{v} \geq i_{\max }\right\},
$$

when each node $a \in \mathcal{A}_{0}$ is transmitting with power $s_{a}$. This threshold can be set to satisfy the interference tolerance policy of the primary system.

The objective of the proposed OSA scheme is to quantify the MIFTP that can be allocated to secondary node $b$. The 
MIFTP for node $b$ is defined as the maximum power that can be allocated to $b$ such that the interference probability with respect to any potential victim node within the coverage distance of a transmitter $p \in \mathcal{P}$ does not exceed a threshold $\varepsilon_{\text {int }}>0, \forall p \in \mathcal{P}$. More formally, the MIFTP for node $b$ with respect to a single transmitter $p$ can be defined as follows [24]:

$s_{b}^{*}(p)=\max \left\{s_{b}: P_{\mathrm{int}}\left(\mathcal{A}_{0} ; s_{b}, x, y\right) \leq \varepsilon_{\mathrm{int}} ; \forall(x, y) \in \bar{B}_{\mathrm{cov}}(p)\right\}$,

where the notation $P_{\text {int }}\left(\mathcal{A}_{0} ; s_{b}, x, y\right)$ is meant to emphasize that the interference probability is a function of node $b$ 's transmit power $s_{b}$ and the location $(x, y)$ of a potential victim node $v$. The MIFTP of node $b$ in the presence of the set of cochannel primary transmitters $\mathcal{P}$ is then given by $s_{b}^{*} \triangleq \min _{p \in \mathcal{P}} s_{b}^{*}(p)$.

\section{T-map}

In a network consisting of multiple cochannel transmitters, the parameter of interest is $\boldsymbol{\Theta} \triangleq\left\{\boldsymbol{\theta}_{p}, \forall p \in \mathcal{P} \cup \mathcal{A}_{T}\right\}$, with $\boldsymbol{\theta}_{p} \triangleq\left(x_{p}, y_{p}, s_{p}\right)$, where $s_{p}$ is the transmit power of node $p$, located at $\left(x_{p}, y_{p}\right)$. It is clear from (6) that in order to compute the MIFTP, it is necessary to estimate $\Theta$. The presence of cochannel interference increases the error in estimating $\Theta$, which can be mitigated if the secondary nodes share their estimates with other more distant secondary nodes (see Section III). Therefore, we propose the maintenance of a distributed database, called the T-map (Transmitter-map), containing relevant information about all cochannel transmitters.

It is important to note that the nodes exchange only SS measurement updates, not entire copies of their local T-maps. Thus, the communication overhead of maintaining the T-map in the network is relatively small. In general, each node may have a different local T-map, which reflects the SS measurement data it has received from other nodes. The local T-map is maintained in a manner analogous to the way a routing table is maintained in a link-state routing protocol. However, unlike a link-state protocol, SS measurement updates need not be propagated throughout the entire network, since the presence of a given cochannel transmitter can be ignored by secondary nodes beyond a distance that depends on the maximum transmit power of secondary nodes and the coverage distance of the given transmitter. A full treatment of the protocols required to maintain the T-map is beyond the scope of the present paper.

In [24], it was shown that given a set of SS measurements, the maximum likelihood (ML) estimator is optimal in the mean square error (MSE) sense and optimality is achieved as the observation noise becomes vanishingly small. The ML estimate (MLE) of the Cramér-Rao Bound (CRB) was found to provide an accurate approximation for the estimation error. Hence, we propose that the T-map store the MLEs of each transmitter's parameters and the associated CRBs:

$$
\boldsymbol{T} \triangleq\left\{\left(\hat{\boldsymbol{\theta}}_{p}, \hat{\boldsymbol{J}}_{\boldsymbol{\theta}_{p}}^{-1}\right), \forall p \in \mathcal{P}\right\} \cup\left\{\boldsymbol{\theta}_{a}, \forall a \in \mathcal{A}_{T}\right\},
$$

where $\hat{\boldsymbol{\theta}}_{p}$ is the $\mathrm{MLE}^{1}$ of $\boldsymbol{\theta}_{p}$ and $\hat{\boldsymbol{J}}_{\boldsymbol{\theta}_{p}}^{-1}$ is the MLE of the associated CRB.

\footnotetext{
${ }^{1}$ Throughout this paper, all estimates indicated by ${ }^{\wedge}$ represent MLEs.
}

In general, the true parameters of some nodes in $\mathcal{A}_{T}$ may not be known. In this case, we treat these particular secondary transmitters as primary transmitters and estimate their corresponding unknown parameters. For a given frequency channel $\nu$ and time $t$, the T-map $\boldsymbol{T}(\nu, t)$ characterizes the spatial region where secondary transmissions can be allowed. For a static set of primary transmitters, the local T-map maintained by a secondary node should converge after a certain time period. In a dynamic scenario, the T-map should track changes that take place in the spectrum occupancy profile over time.

\section{Collaborative Sensing Scheme}

In our proposed collaborative sensing scheme, the secondary nodes estimate the MIFTP given the information on the cochannel transmitters contained in the T-map. To estimate the MIFTP, the secondary nodes must first update the T-map from their received SS measurements. This SS observation set is denoted by $\mathcal{O} \triangleq\left\{\left(R_{a}, \boldsymbol{L}_{a}\right): a \in \mathcal{A}\right\}$, where $R_{a}$ is the net SS received due to all cochannel transmitters at the secondary node $a$, located at $\boldsymbol{L}_{a} \triangleq\left(x_{a} y_{a}\right)$. Cochannel interference due to the primary transmitters introduces error in the SS measurements. For example, to localize $p \in \mathcal{P}$, instead of $\left\{R_{a p}: a \in \mathcal{A}\right\}$, only $\left\{R_{a}: a \in \mathcal{A}\right\}$ can be observed, resulting in higher estimation error. This effect can be mitigated by the common knowledge of location estimates of the interfering primary transmitters among the secondary nodes maintained via the T-map construct and by accounting for the associated cochannel interference. In the remainder of this section, we consider the case $M=2$. Generalization of the approach to arbitrary $M$ is straightforward.

\section{A. With no information}

Given a set of independent local observations $\mathcal{O}_{1} \triangleq$ $\left\{\left(R_{a}, \boldsymbol{L}_{a}\right): a \in \mathcal{A}_{1} \subset \mathcal{A}\right\}$ in the vicinity of a primary transmitter, say $p_{1} \in \mathcal{P}$, the MLE of the parameter $\boldsymbol{\theta}_{1} \triangleq$ $\left[\begin{array}{lll}x_{p_{1}} & y_{p_{1}} & s_{p_{1}}\end{array}\right]^{T}$ can be found. In the absence of any information about other cochannel transmitters, the log-likelihood function has the following form [24]:

$$
F_{1 A}\left(\boldsymbol{\theta}_{1}\right) \triangleq \sum_{a \in \mathcal{A}_{1}} \ln f_{R_{a} \mid \boldsymbol{\theta}_{1}}
$$

where $R_{a} \mid \boldsymbol{\theta}_{1} \sim \mathcal{N}\left(s_{p_{1}}-g\left(d_{a p_{1}}\right), \sigma_{W}^{2}\right)$. The MLE is found by solving the optimization problem $\hat{\boldsymbol{\theta}}_{1 A}=\arg \max _{\boldsymbol{\theta}_{1}} F_{1 A}\left(\boldsymbol{\theta}_{1}\right)$.

\section{B. With true information}

If the true parameter $\boldsymbol{\theta}_{2} \triangleq\left[\begin{array}{lll}x_{p_{2}} & y_{p_{2}} & s_{p_{2}}\end{array}\right]^{T}$, of another cochannel transmitter $p_{2}$, is known, the observations in $\mathcal{O}_{1}$ can be modeled as

$R_{a}=10 \log _{10}\left(10^{\frac{R_{a p_{1}}}{10}}+10^{\frac{R_{a p_{2}}}{10}}\right)=\kappa^{-1} \ln \left(e^{\kappa R_{a p_{1}}}+e^{\kappa R_{a p_{2}}}\right)$,

where $\kappa \triangleq \frac{\ln 10}{10}$. Approximating the sum of independent lognormal random variables by another lognormal [26], yields 


$$
\begin{aligned}
R_{a} \mid \boldsymbol{\theta}_{1}, \boldsymbol{\theta}_{2} \sim \mathcal{N}\left(\frac{\mu_{B a}}{\kappa}, \frac{\sigma_{B a}^{2}}{\kappa^{2}}\right), \text { where } \\
\mu_{B a} \triangleq \ln \left(k_{1}\right)-\frac{\sigma_{B a}^{2}}{2}, \quad \sigma_{B a}^{2} \triangleq \ln \left(1+\frac{k_{2}^{2}}{k_{1}^{2}}\right), \\
k_{1} \triangleq e^{\frac{\kappa^{2} \sigma_{W}^{2}}{2}}\left(e^{\kappa u_{a p_{1}}}+e^{\kappa u_{a p_{2}}}\right), \\
k_{2}^{2} \triangleq e^{\kappa^{2} \sigma_{W}^{2}}\left(e^{\kappa^{2} \sigma_{W}^{2}}-1\right)\left(e^{2 \kappa u_{a p_{1}}}+e^{2 \kappa u_{a p_{2}}}\right),
\end{aligned}
$$

and $u_{i j} \triangleq s_{j}-g\left(d_{i j}\right)$. Note that $u_{a p_{2}}$ is known and $\boldsymbol{\theta}_{1}$ is the only unknown. The log-likelihood function is $F_{1 B}\left(\boldsymbol{\theta}_{1}\right) \triangleq \sum_{a \in \mathcal{A}_{1}} \ln f_{R_{a} \mid \boldsymbol{\theta}_{1}, \boldsymbol{\theta}_{2}}$, with ML solution $\hat{\boldsymbol{\theta}}_{1 B}\left(\boldsymbol{\theta}_{1}\right)=$ $\arg \max _{\boldsymbol{\theta}_{1}} F_{1 B}\left(\boldsymbol{\theta}_{1}\right)$.

We have observed (see Fig. 1) that in the region of practical interest, $u_{a p_{i}} \in[-150,100] \mathrm{dBm}, \sigma_{B a} \lesssim \kappa \sigma_{W}, \forall a$, where $x_{1} \lesssim x_{2}$ means that $x_{1}$ is upper bounded by $x_{2}$ which is not too far from $x_{1}$. If the observations $R_{a}$ are scaled as $\widetilde{R}_{a}=$ $\kappa R_{a}$, this approximation can be used to obtain an equivalent but simpler objective function compared to $F_{1 B}\left(\boldsymbol{\theta}_{1}\right)$. In this case, we have $\hat{\boldsymbol{\theta}}_{1 B}=\arg \max _{\boldsymbol{\theta}_{1}} \widetilde{F}_{1 B}\left(\boldsymbol{\theta}_{1}\right)$, where

$$
\widetilde{F}_{1 B}\left(\boldsymbol{\theta}_{1}\right) \triangleq \sum_{a \in \mathcal{A}_{1}} \ln f_{\widetilde{R}_{a} \mid \boldsymbol{\theta}_{1}, \boldsymbol{\theta}_{2}}
$$

and $\widetilde{R}_{a} \mid \boldsymbol{\theta}_{1}, \boldsymbol{\theta}_{2} \sim \mathcal{N}\left(\ln \left(\sum_{i=1,2} e^{\kappa u_{a p_{i}}}\right), \kappa^{2} \sigma_{W}^{2}\right)$.

\section{With estimated information}

In many cases, only the estimated information about other cochannel transmitters is available, via the distributed maintenance of the T-map. Assume that the ML estimated parameters $\left(\hat{\boldsymbol{\theta}}_{2}, \hat{\boldsymbol{J}}_{\boldsymbol{\theta}_{2}}^{-1}\right)$ of transmitter $p_{2}$ are known. Note that here $\hat{\boldsymbol{\theta}}_{2}$ is found by solving the likelihood function $F_{2 A}$ corresponding to $p_{2}$ (similar to $F_{1 A}$ ), i.e., $\hat{\boldsymbol{\theta}}_{2} \equiv \hat{\boldsymbol{\theta}}_{2 A}$ and $\hat{\boldsymbol{J}}_{\boldsymbol{\theta}_{2}}^{-1} \equiv \hat{\boldsymbol{J}}_{\boldsymbol{\theta}_{2 A}}^{-1}$. Instead of $u_{a p_{2}}$, we can obtain $\hat{u}_{a p_{2}}$, where $\hat{u}_{a p_{2}} \triangleq \hat{S}_{p_{2}}-g\left(\hat{D}_{a p_{2}}\right)$ denotes the MLE of $u_{a p_{2}}$ and $\hat{\boldsymbol{\theta}}_{2}=\left[\begin{array}{lll}\hat{X}_{p_{2}} & \hat{Y}_{p_{2}} & \hat{S}_{p_{2}}\end{array}\right]^{T}$ denotes the MLE of $\boldsymbol{\theta}_{2}$, via the invariance principle (cf. [27, p. 217]), which states that the MLE of a function $q(\cdot)$ of $\boldsymbol{\Phi}$ is given by $q(\hat{\boldsymbol{\Phi}})$, where $\hat{\boldsymbol{\Phi}}$ denotes the MLE of $\boldsymbol{\Phi}$. Since the MLE of the $\mathrm{CRB}$ approaches the estimation error as $\sigma_{W} \rightarrow 0$ [24], $R_{a p_{2}}$ can be modeled as follows:

$$
R_{a p_{2}}=u_{a p_{2}}+W_{a p_{2}}=\hat{u}_{a p_{2}}+W_{2 a}+W_{a p_{2}},
$$

where $W_{2 a} \sim \mathcal{N}\left(0, \hat{\sigma}_{2 a}^{2}\right)$ with $\hat{\sigma}_{2 a}^{2} \triangleq \hat{\boldsymbol{H}}_{a}^{T} \hat{\boldsymbol{J}}_{\boldsymbol{\theta}_{2}}^{-1} \hat{\boldsymbol{H}}_{a}$. Again, $\hat{\boldsymbol{H}}_{a}$ is the MLE of $\boldsymbol{H}_{a}$ where

$$
\begin{aligned}
\boldsymbol{H}_{a} & \triangleq\left[\frac{\partial u_{a p_{2}}}{\partial x_{p_{2}}}, \frac{\partial u_{a p_{2}}}{\partial y_{p_{2}}}, \frac{\partial u_{a p_{2}}}{\partial s_{p_{2}}}\right]^{T} \\
& =\left[-\dot{g}\left(d_{a p_{2}}\right) \cos \phi_{a p_{2}},-\dot{g}\left(d_{a p_{2}}\right) \sin \phi_{a p_{2}}, 1\right]^{T},
\end{aligned}
$$

and $\dot{g}(d) \triangleq \frac{\partial g(d)}{\partial d}$. Hence, $R_{a p_{2}} \mid \hat{\boldsymbol{\theta}}_{2} \sim \mathcal{N}\left(\hat{u}_{a p_{2}}, \hat{\sigma}_{2 a}^{2}+\sigma_{W}^{2}\right)$ and $R_{a} \mid \boldsymbol{\theta}_{1}, \hat{\boldsymbol{\theta}}_{2} \sim \mathcal{N}\left(\frac{\mu_{C a}}{\kappa}, \frac{\sigma_{C a}^{2}}{\kappa^{2}}\right)$, where

$$
\begin{aligned}
\mu_{C a} \triangleq & \ln \left(k_{3}\right)-\frac{\sigma_{C a}^{2}}{2}, \quad \sigma_{C a}^{2} \triangleq \ln \left(1+\frac{k_{4}^{2}}{k_{3}^{2}}\right), \\
k_{3} \triangleq & e^{\frac{\kappa^{2} \sigma_{W}^{2}}{2}}\left(e^{\kappa u_{a p_{1}}}+e^{\kappa \hat{u}_{a p_{2}}+\frac{\kappa^{2} \hat{\sigma}_{2 a}^{2}}{2}}\right), \\
k_{4}^{2} \triangleq & \left(e^{\kappa^{2} \sigma_{W}^{2}}-1\right) e^{2 \kappa u_{a p_{1}}+\kappa^{2} \sigma_{W}^{2}} \\
& +\left(e^{\kappa^{2} \hat{\sigma}_{2 a}^{2}+\kappa^{2} \sigma_{W}^{2}}-1\right) e^{2 \kappa \hat{u}_{a p_{2}}+\kappa^{2} \hat{\sigma}_{2 a}^{2}+\kappa^{2} \sigma_{W}^{2}} .
\end{aligned}
$$

The corresponding log-likelihood function is $F_{1 C}\left(\boldsymbol{\theta}_{1}\right) \triangleq$ $\sum_{a \in \mathcal{A}_{1}} \ln f_{R_{a} \mid \boldsymbol{\theta}_{1}, \hat{\boldsymbol{\theta}}_{2}}$, and the ML solution is given by $\hat{\boldsymbol{\theta}}_{1 C}=$ $\arg \max _{\boldsymbol{\theta}_{1}} F_{1 C}\left(\boldsymbol{\theta}_{1}\right)$. Similar to Section III-B, to simplify the objective function we can use the scaled observations to solve $\hat{\boldsymbol{\theta}}_{1 C}=\arg \max _{\boldsymbol{\theta}_{1}} \widetilde{F}_{1 C}\left(\boldsymbol{\theta}_{1}\right)$, where

$$
\widetilde{F}_{1 C}\left(\boldsymbol{\theta}_{1}\right) \triangleq \sum_{a \in \mathcal{A}_{1}} \ln f_{\widetilde{R}_{a} \mid \boldsymbol{\theta}_{1}, \hat{\boldsymbol{\theta}}_{2}},
$$

and $\widetilde{R}_{a} \mid \boldsymbol{\theta}_{1}, \hat{\boldsymbol{\theta}}_{2} \sim \mathcal{N}\left(\ln \left(e^{\kappa u_{a p_{1}}}+e^{\kappa \hat{u}_{a p_{2}}+\frac{\kappa^{2} \hat{\sigma}_{2 a}^{2}}{2}}\right), \kappa^{2} \sigma_{W}^{2}\right)$. Note that $\hat{\boldsymbol{\theta}}_{1 C} \rightarrow \hat{\boldsymbol{\theta}}_{1 B}$, as $\sigma_{2 a} \rightarrow 0, \forall a$. Our hypothesis is that $\hat{\boldsymbol{\theta}}_{1 B}$ and $\hat{\boldsymbol{\theta}}_{1 C}$ are better estimators than $\hat{\boldsymbol{\theta}}_{1 A}$ in terms of mitigating the error induced by cochannel interference ${ }^{2}$. The effectiveness of this proposed collaborative sensing strategy is studied numerically in Section V.

\section{Measurement clustering}

When multiple cochannel transmitters are present, accurate localization depends on using an appropriate set of SS measurements. More precisely, as stated in Section III, the measurements should be shared locally among neighboring CRs. For locating a particular transmitter, the most useful measurements are received by nodes residing in its vicinity. This is because the effect of cochannel interference on these measurements is expected to be small. On the other hand, the worst measurements are the ones which have equal contributions of received power from multiple transmitters. Since it is difficult to resolve the power contribution from each transmitter, a large error in localization can be incurred in this case. Thus, it is important to collect measurements that have the strongest contribution from a particular transmitter. This is equivalent to assigning each measurement to the transmitter closest to it. Therefore, to minimize the effect of cochannel interference, all the measurements should be clustered appropriately, where each measurement cluster represents the subset of measurements to be used in the localization of a particular transmitter.

In [29], two schemes for measurement clustering, one based on minimum description length (cf. [30]) and the other based on minimum MSE, are proposed. Both schemes produce an estimate, $\hat{M}$, of the number of cochannel transmitters, together with an associated set of initial parameter estimates, $\left\{\hat{\boldsymbol{\theta}}_{i}\right\}_{i=1}^{\hat{M}}$, which is most likely to have generated the given set of measurements. The measurements are then assigned to the nearest estimated transmitter from $\left\{\hat{\boldsymbol{\theta}}_{i}\right\}_{i=1}^{\hat{M}}$. Once the initial estimates are found via measurement clustering, the effect of cochannel interference can be mitigated using the approach discussed in Section III-C. In particular, note that $\hat{\boldsymbol{\theta}}_{1 C}$ is a better estimator than $\hat{\boldsymbol{\theta}}_{1 A}$ (in terms of MSE), since it uses the information of $\hat{\boldsymbol{\theta}}_{2}$. Symbolically, we denote this by $\hat{\boldsymbol{\theta}}_{1 A} \stackrel{\hat{\boldsymbol{\theta}}_{2}}{\rightarrow} \hat{\boldsymbol{\theta}}_{1 C}$. The corresponding compensation for $\hat{\boldsymbol{\theta}}_{2}$ is given by $\hat{\boldsymbol{\theta}}_{2} \stackrel{\hat{\boldsymbol{\theta}}_{1 C}}{\rightarrow} \hat{\boldsymbol{\theta}}_{2 C}$, where $\hat{\boldsymbol{\theta}}_{2 C}$ denotes the modified estimator of $\hat{\boldsymbol{\theta}}_{2}$ incorporating the knowledge of $\hat{\boldsymbol{\theta}}_{1 C}$. We can continue the procedure as $\hat{\boldsymbol{\theta}}_{1 C} \stackrel{\hat{\boldsymbol{\theta}}_{2 C}}{\rightarrow} \hat{\boldsymbol{\theta}}_{1 C}^{\prime}$, then $\hat{\boldsymbol{\theta}}_{2 C} \stackrel{\hat{\boldsymbol{\theta}}_{1 C}^{\prime}}{\rightarrow} \hat{\boldsymbol{\theta}}_{2 C}^{\prime}$, and so

\footnotetext{
${ }^{2}$ CRBs corresponding to $\hat{\boldsymbol{\theta}}_{1 B}$ and $\hat{\boldsymbol{\theta}}_{1 C}$ for arbitrary $M$ are derived in [28].
} 
on. A simple convergence criterion should be used to halt the recursive procedure, providing a suitable tradeoff between accuracy and computational load. For example, the rule may simply be to stop when the difference between successive iterations is sufficiently small.

\section{E. Impact of infrequent measurement updates}

If SS measurement updates arrive infrequently to a node due to link failures, a given node will not be able to compute the most up-to-date primary transmitter location estimates for its local T-map. The infrequent arrival of measurement updates may lead to larger errors in the primary transmitter location estimates, which will in turn propagate to the associated MIFTP estimates. The MIFTP approximation method presented in Section IV tends to be more conservative when only a small number of measurements is available (cf. Section V). If fewer than three measurements are available and hence a location estimate cannot be computed, the proposed scheme defaults to Listen-Before-Talk (LBT) (cf. [23]). In the LBT scheme, the secondary node is allowed to transmit with low power only for short time intervals when it senses the channel to be idle. Thus, the impact of infrequent measurement updates is that the transmission power used by the node may be overly restricted, reducing its potential transmission capacity. In general though, the proposed collaborative sensing scheme should perform much better than the LBT scheme, provided a sufficient number of SS measurements is available at a node to compute the location estimates.

\section{Maximum Interference-Free Transmit Power}

In this section, we first formulate an approach to compute the true MIFTP for a secondary node $b \in \mathcal{A} \backslash \mathcal{A}_{T}$ (see (6)) and then develop a practical approximation for the MIFTP. For brevity, we omit detailed proofs, but they may be found in [28].

\section{A. True MIFTP calculation}

The interference probability can be expressed as follows.

Proposition 1: The interference probability at victim node $v$ due to nodes in $\mathcal{A}_{0}$ is given by

$$
P_{\text {int }}\left(\mathcal{A}_{0} ; s_{b}, v\right)=Q\left(\frac{\kappa i_{\max }-\mu}{\sigma}\right),
$$

where

$$
\begin{aligned}
& \mu \triangleq \frac{\kappa^{2} \sigma_{W}^{2}}{2}-\frac{\sigma^{2}}{2}+h, \sigma^{2} \triangleq \ln \left(1+\frac{k_{6}^{2}}{k_{5}^{2}}\right), k_{5} \triangleq e^{\frac{\kappa^{2} \sigma_{W}^{2}}{2}+h}, \\
& h \triangleq \ln \left(L_{b}(v)+\sum_{a \in \mathcal{A}_{T}} L_{a}(v)\right), \\
& k_{6}^{2} \triangleq e^{\kappa^{2} \sigma_{W}^{2}}\left(e^{\kappa^{2} \sigma_{W}^{2}}-1\right)\left(L_{b}^{2}(v)+\sum_{a \in \mathcal{A}_{T}} L_{a}^{2}(v)\right)
\end{aligned}
$$

and $L_{a}(v) \triangleq e^{\kappa\left(s_{a}-g\left(d_{v a}\right)\right)}$ for any secondary node $a \in \mathcal{A}_{0}$.

The interference probability at victim $v$ depends on three quantities: (i) the interference tolerance threshold, $i_{\max }$, (ii) the variance of the shadowing noise, $\sigma_{W}^{2}$, and (iii) the aggregate interference power, $h$, received at $v$. The following lemma provides a method for computing the MIFTP.

Lemma 1: For a secondary node $b$ such that $\left(x_{b}, y_{b}\right) \notin$ $\bar{B}_{\text {cov }}(p)$ the MIFTP with respect to primary transmitter $p \in \mathcal{P}$ is given by

$$
s_{b}^{*}(p)=\min _{(x, y) \in \bar{B}_{\mathrm{cov}}(p)} s_{b}^{*}(p ; x, y)
$$

where

$$
s_{b}^{*}(p ; x, y) \triangleq \max \left\{s_{b}: P_{\text {int }}\left(\mathcal{A}_{0} ; s_{b}, x, y\right) \leq \varepsilon_{\text {int }}\right\} .
$$

The complexity of the optimization problem suggested by Lemma 1 can be reduced by restricting the minimization problem to the boundary of the coverage region $\bar{B}_{\text {cov }}(p)$ as stated in the next proposition.

Proposition 2: Given a set of secondary cochannel transmitters with parameters $\left\{\boldsymbol{\theta}_{a}\right\}=\left\{\left(x_{a}, y_{a}, s_{a}\right)\right\}$, all located outside the coverage region $\bar{B}_{\text {cov }}(p)$, the maximum interference due to path loss alone is achieved on the boundary $\partial B_{\operatorname{cov}}(p)$, i.e., the circle centered at $p$ with radius $d_{\text {cov }}(p)$.

Combining Lemma 1 and Proposition 2 simplifies the computation of MIFTP.

Corollary 1:

$$
\begin{aligned}
s_{b}^{*}(p) & =\min _{(x, y) \in \partial B_{\mathrm{cov}}(p)} s_{b}^{*}(p ; x, y) \\
& =\min _{\psi \in[0,2 \pi)} s_{b}^{*}\left(p ; x_{p}(\psi), y_{p}(\psi)\right)
\end{aligned}
$$

with $s_{b}^{*}(p ; x, y)$ defined in (19), and

$$
x_{p}(\psi) \triangleq x_{p}+d_{\text {cov }}(p) \cos \psi, y_{p}(\psi) \triangleq y_{p}+d_{\text {cov }}(p) \sin \psi \text {. }
$$

The true MIFTP as defined in Lemma 1 or Corollary 1 cannot be calculated directly, since the T-map provides only $\left\{\hat{\boldsymbol{\theta}}_{p}, \hat{\boldsymbol{J}}_{\boldsymbol{\theta}_{p}}^{-1}: p \in \mathcal{P}\right\}$ and $\left\{\boldsymbol{\theta}_{a}: a \in \mathcal{A}_{T}\right\}$. Therefore, we develop an approximation to the MIFTP of $b$ with respect to $p \in \mathcal{P}$ by first estimating the critical distance, $\hat{D}_{b}^{*}(p)$ to detect the presence of a spectrum hole. Then, an estimate, $\hat{s}_{b}^{*}(p)$, for the MIFTP is obtained by considering potential victim nodes lying on the circle $\partial B_{\operatorname{cov}}(p)$.

\section{B. Spectrum hole detection}

For a particular $p \in \mathcal{P}$, the critical distance estimate with respect to node $b$ is given by

$$
\begin{aligned}
\hat{D}_{b}(p) \triangleq & \hat{D}_{p b}-\hat{D}_{\mathrm{cov}}(p) \\
\stackrel{(3)}{=} & \sqrt{\left(\hat{X}_{p}-x_{b}\right)^{2}+\left(\hat{Y}_{p}-y_{b}\right)^{2}}-g^{-1}\left(\hat{S}_{p}-r_{\min }\right. \\
& \left.+\sigma_{W} Q^{-1}\left(1-\varepsilon_{\mathrm{cov}}\right)\right),
\end{aligned}
$$

where $\hat{D}_{p b}$ and $\hat{D}_{\text {cov }}(p)$ denote the MLEs of $d_{p b}$ and $d_{\text {cov }}(p)$, respectively. In the asymptotic regime $\sigma_{W} \rightarrow 0$,

$$
E_{p} \triangleq \hat{D}_{b}(p)-d_{b}(p)=\hat{D}_{p b}-d_{p b}-\left(\hat{D}_{\mathrm{cov}}(p)-d_{\mathrm{cov}}(p)\right)
$$

can be modeled as $E_{p} \sim \mathcal{N}\left(0, \hat{J}_{p b}^{-1}\right)$, where $\hat{J}_{p b}^{-1}$ denotes the MLE of the CRB corresponding to the error in estimating $\hat{D}_{b}(p)$, [24, Proposition 6].

Suppose $E_{p}=r$ and $\hat{D}_{b}(p)=r_{0}$. If $|r| \geq r_{0}>0$, then in the worst case, node $b$ lies within $d_{\text {cov }}(p)$ of primary transmitter $p$. In this scenario, node $b$ must not transmit, i.e., $s_{b}^{*}=-\infty$, to avoid potentially harmful interference to the 
victim nodes. If $0<|r|<r_{0}$, then $b$ can transmit, i.e., $s_{b}^{*} \neq-\infty$. Since we do not know $r$, we can only ensure that for the given realization $\hat{D}_{b}(p)=r_{0}>0$, the event $\left\{\left|E_{p}\right|<r_{0}\right\}$ occurs with high probability. In particular, for $s_{b}^{*} \neq-\infty$ and $\varepsilon \in(0,1)$, we require $r_{0}>\hat{R}^{*}>0$, where

$R^{*} \triangleq \min \left\{R: \operatorname{Pr}\left(\left|E_{p}\right|<R\right) \geq \varepsilon\right\}=\sqrt{\hat{J}_{p b}^{-1}} \cdot Q^{-1}\left(\frac{1-\varepsilon}{2}\right)$.

For example, for $\varepsilon=0.9973, \hat{R}^{*} \approx 3 \sqrt{\hat{J}_{p b}^{-1}}$. Define the set $\mathcal{D} \triangleq\left\{p \in \mathcal{P}: \hat{D}_{b}(p) \leq \hat{R}^{*}\right\}$. Whenever, $\mathcal{D}=\emptyset$, a spectrum hole with respect to $b$ is detected, and the approximate MIFTP, $\hat{s}_{b}^{*}$ should be computed.

\section{Interference probability and MIFTP approximation}

An upper bound on $P_{\text {int }}\left(\mathcal{A}_{0} ; s_{b}, v\right)$ is given as follows.

Proposition 3: The interference probability $P_{\text {int }}\left(\mathcal{A}_{0} ; s_{b}, v\right)$ at a particular victim $v$ located at $\left(x_{v}, y_{v}\right)$ can be upper bounded by $Q(\gamma)$, where $\gamma \triangleq \frac{\kappa i_{\max }-\frac{\kappa^{2} \sigma_{W}^{2}}{2}+\frac{\sigma^{2}}{2}-h}{\kappa \sigma_{W}}$ (cf. (16) and (17)).

Define $F(\gamma) \triangleq \hat{\Gamma}-\gamma$, where $\hat{\Gamma}$ denotes the MLE of $\gamma$. Note that $\gamma$ is a function of $\boldsymbol{\theta}_{p}$. Under some regularity conditions [27, p. 229], the CRBs of $\boldsymbol{\theta}_{p}$ and $\gamma$ are related as $J_{\gamma}^{-1}=\boldsymbol{H}_{0}^{T} \boldsymbol{J}_{\boldsymbol{\theta}_{p}}^{-1} \boldsymbol{H}_{0}$, where $\boldsymbol{H}_{0} \triangleq\left[\frac{\partial \gamma}{\partial x_{p}} \frac{\partial \gamma}{\partial y_{p}} \frac{\partial \gamma}{\partial s_{p}}\right]^{T}$ and is evaluated in [28]. In [24], we provide a closed-form expression for $\boldsymbol{J}_{\boldsymbol{\theta}_{p}}^{-1}$ and show that it is achievable as $\sigma_{W} \rightarrow 0$. It can be shown that if $\boldsymbol{J}_{\boldsymbol{\theta}_{p}}^{-1}$ is achievable asymptotically as $\sigma_{W} \rightarrow 0$, then so is $J_{\gamma}^{-1}$, (cf. [24, Proposition 4]). This means that in the asymptotic regime $F(\gamma) \sim \mathcal{N}\left(0, J_{\gamma}^{-1}\right)$. Suppose for a particular realization that $F(\gamma)=x$ and $\hat{\Gamma}=\hat{\gamma}$. Then, the upper bound on the interference probability conditioned on $F(\gamma)=x$ is given by $Q(\hat{\gamma}-x)$. Using the total probability theorem

$$
\begin{gathered}
\int_{-\infty}^{\infty} Q(\hat{\gamma}-x) \mathcal{N}\left(0, J_{\gamma}^{-1}\right) d x=Q\left(\frac{\hat{\gamma}}{\sqrt{1+J_{\gamma}^{-1}}}\right) \\
\leq \frac{1}{2} e^{-\frac{\hat{\gamma}^{2}}{2\left(1+J_{\gamma}^{-1}\right)}} \triangleq w\left(s_{b}, x, y\right),
\end{gathered}
$$

where the first equality is obtained using a result in [31, p. 102] and the upper bound is valid for $\hat{\gamma} \geq 0$. We propose to approximate the MIFTP in terms of this upper bound ${ }^{3} w$ on the interference probability averaged over all possible estimation errors. Since $J_{\gamma}^{-1}$ is unknown, using the invariance principle we replace it by its MLE, $\hat{J}_{\gamma}^{-1}$, and denote the expression corresponding to $(23)$ by $\hat{w}\left(s_{b}, x, y\right)$. In a manner analogous to Corollary 1, an approximation to the MIFTP can be computed as follows:

$$
\hat{s}_{b}^{*}(p) \triangleq \min _{\psi \in[0,2 \pi)} \hat{s}_{b}^{*}\left(p ; \hat{X}_{p}(\psi), \hat{Y}_{p}(\psi)\right),
$$

where

$$
\begin{aligned}
& \hat{X}_{p}(\psi) \triangleq \hat{X}_{p}+\hat{D}_{\mathrm{cov}}(p) \cos \psi, \hat{Y}_{p}(\psi) \triangleq \hat{Y}_{p}+\hat{D}_{\mathrm{cov}}(p) \sin \psi, \\
& \hat{s}_{b}^{*}(p ; x, y) \triangleq \max \left\{s_{b}: \hat{w}\left(s_{b}, x, y\right) \leq \varepsilon_{\mathrm{int}}\right\} .
\end{aligned}
$$

\footnotetext{
${ }^{3}$ If, for any realization $\hat{\gamma}<0$, we can use (22) to compute the MIFTP.
}

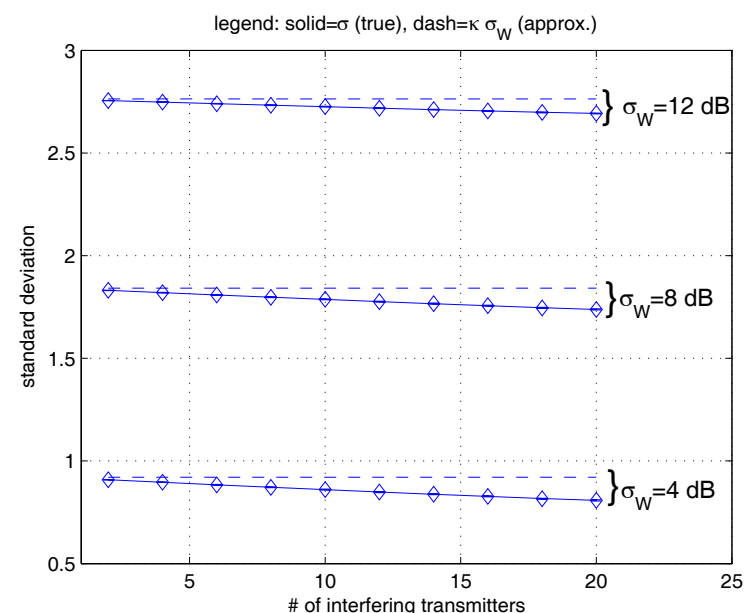

Fig. 1. Plot shows the mean values of $\sigma_{B a}$, calculated according to (9)-(11), with the associated $99.7 \%$ confidence intervals generated from $10^{4}$ random realizations of $u_{a p_{i}} \in[-150,100] \mathrm{dB}, \forall p_{i} \in \mathcal{P}$, where $|\mathcal{P}| \in[2,20]$.

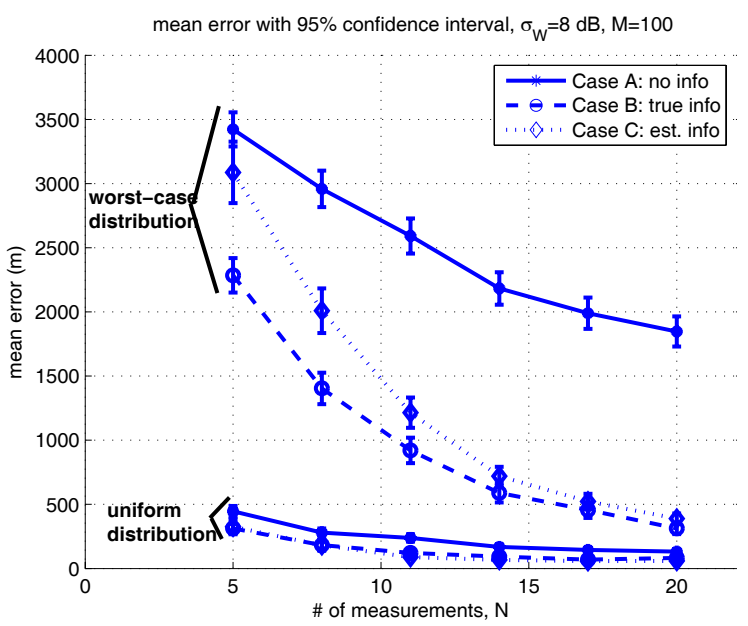

Fig. 2. Localization error $\mathcal{E}_{1}$ vs. number of measurements.

A computationally simpler approximation to MIFTP can be obtained by assuming that the worst-case victim, say $v^{*}$, lies at the intersection of the circle $\partial B_{\operatorname{cov}}(p)$ and the straight line connecting $\left(x_{b}, y_{b}\right)$ and $\left(\hat{X}_{p}, \hat{Y}_{p}\right)$ :

$$
\hat{s}_{b}^{*}(p)=\max \left\{s_{b}: \hat{w}\left(s_{b}, x_{v^{*}}, y_{v^{*}}\right) \leq \varepsilon_{\text {int }}\right\} .
$$

Numerical results presented in Section $\mathrm{V}$ suggest that this approximation is sufficiently accurate for practical scenarios. We remark that localization accuracy is incorporated into the above MIFTP approximations via the CRB term, $J_{\gamma}^{-1}$. In particular, as the estimation error increases, the MIFTP becomes more conservative, ensuring that the interference tolerance threshold, $i_{\max }$ is met, but also making the OSA scheme less efficient. This property of being conservative is important since secondary transmissions should do no harm to the primary system.

\section{NUMERICAL RESULTS}

For the numerical results presented in this section, we choose system parameter values that reflect the application 


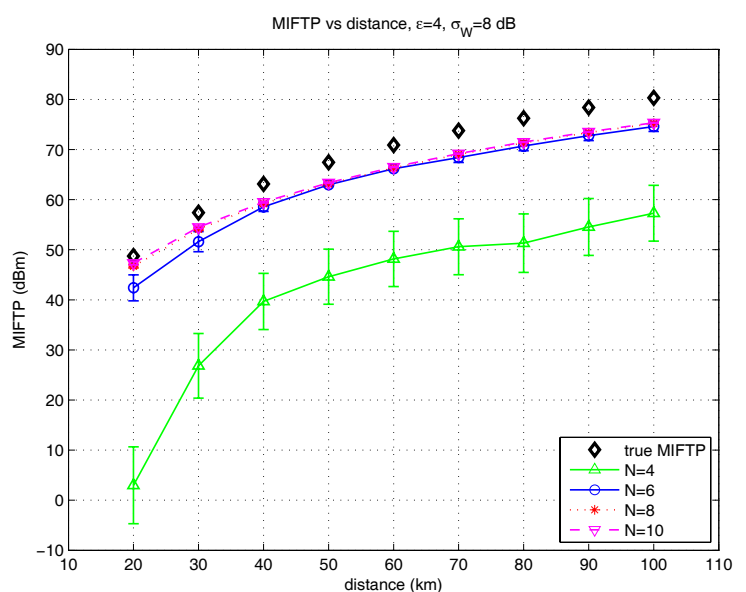

Fig. 3. MIFTP vs. $d_{b p}$, for single primary transmitter $p$.

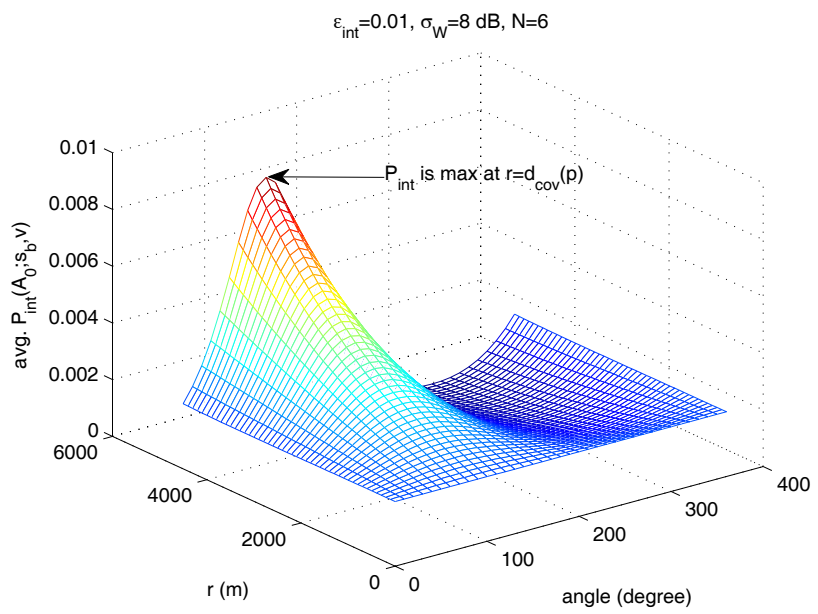

Fig. 4. Average interference probability surface due to $\hat{s}_{b}^{*}(p)$ in the coverage region $\bar{B}_{\operatorname{cov}}(p)$.

of OSA to digital TV broadcast bands. The SS measurements are generated using the generic path loss function $g(d)=10 \epsilon \log _{10}(d)$, where $d$ is distance and $\epsilon$ is the path loss exponent. Unless otherwise specified, all simulations are performed with the following parameter values: detection threshold for victims $r_{\min }=-85 \mathrm{dBm}$, detection threshold for secondary nodes $r_{a}=-90 \mathrm{dBm}$, interference tolerance threshold $i_{\max }=-100 \mathrm{dBm}$, outage probability upper limit $\varepsilon_{\mathrm{cov}}=0.01$, allowable interference probability upper limit to victims $\varepsilon_{\text {int }}=0.01$, shadowing standard deviation $\sigma_{W}=8 \mathrm{~dB}$ and path loss exponent $\epsilon=4$. For a particular primary transmitter $p$ and for each simulation trial, we randomly place $N$ secondary nodes, with uniform distribution inside the coverage region $\bar{B}_{\text {cov }}(p)$. These nodes perform localization of $p$ by evaluating the MLEs $\left(\hat{\boldsymbol{\theta}}_{p}, \hat{\boldsymbol{J}}_{\boldsymbol{\theta}_{p}}^{-1}\right)$ (cf. [24]). Each result is averaged over $K$ trials and shown with the associated $95 \%$ confidence interval, which arises due to randomness in the localizing node positions, as well as the shadowing noise.

\section{A. Mitigation of cochannel interference}

Consider two cochannel primary transmitters $p_{1}$ and $p_{2}$ parameterized by $(8,0,80)$ and $(0,0,80)$, respectively, where the

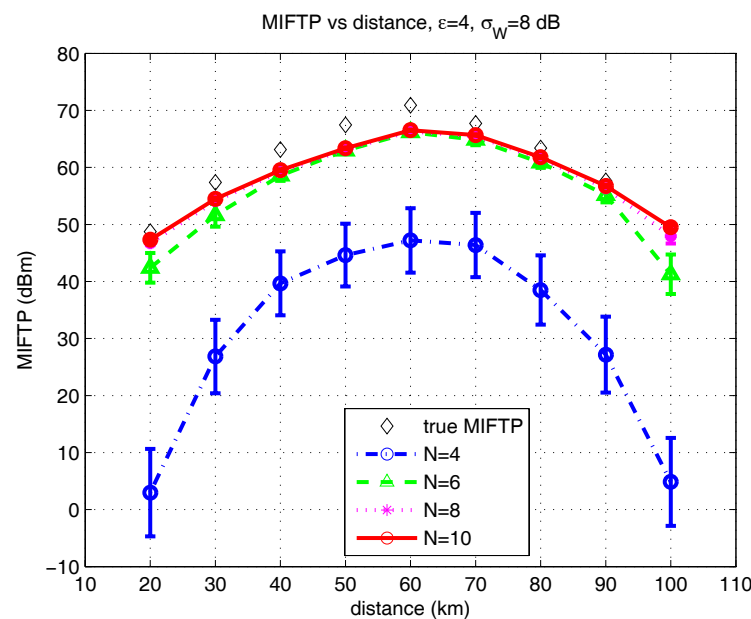

Fig. 5. MIFTP vs. $d_{b p}$, for two primary transmitters, $p$ and $p^{\prime}$.

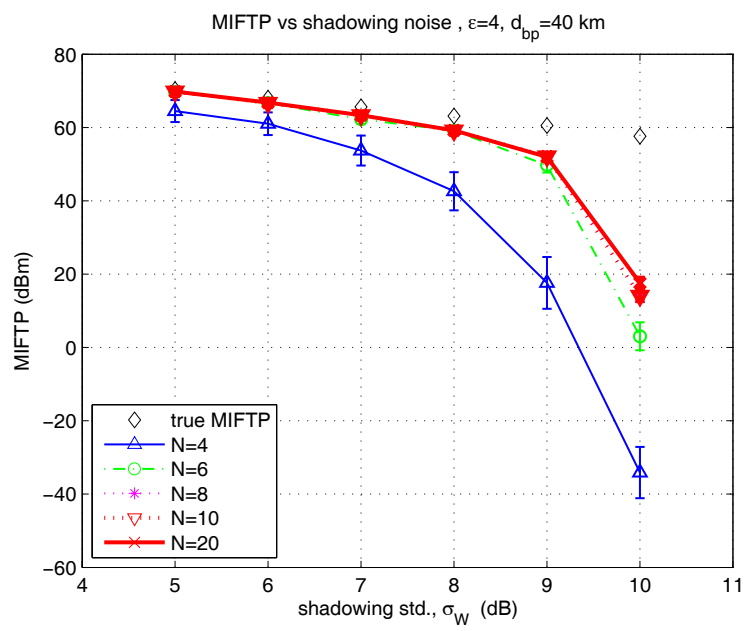

Fig. 6. MIFTP vs. shadowing noise, $\sigma_{W}$.

3-tuple indicates the location and transmit power, with units of $[\mathrm{km}, \mathrm{km}, \mathrm{dBm}]$. For these parameter values, $d_{\text {cov }}\left(p_{1}\right)=$ $d_{\text {cov }}\left(p_{2}\right)=4.6 \mathrm{~km}$ and $d_{\operatorname{det}}\left(p_{1}\right)=d_{\operatorname{det}}\left(p_{2}\right)=6.1 \mathrm{~km}$, (cf. (3) and (4)). Each measurement is generated by averaging over 100 raw measurements to reduce the effect of shadowing noise. We are interested in estimating $\boldsymbol{\theta}_{1}=\left[\begin{array}{lll}x_{p_{1}} & y_{p_{1}} & s_{p_{1}}\end{array}\right]^{T}$. To evaluate the performance of our proposed scheme, we find the ML solutions of the likelihood functions $F_{1 A}, \widetilde{F}_{1 B}$ and $\widetilde{F}_{1 C}$ corresponding to (8), (12) and (13), respectively. As a performance measure, we calculate the mean missed distance:

$$
\mathcal{E}_{1} \triangleq \frac{1}{K} \sum_{i=1}^{K} \sqrt{\left(\hat{X}_{p_{1}}(i)-x_{p_{1}}\right)^{2}+\left(\hat{Y}_{p_{1}}(i)-y_{p_{1}}\right)^{2}},
$$

over $K=1000$ independent trials. In Fig. 2 , we plot $\mathcal{E}_{1}$ as a function of the number of measurements. The bottom three curves correspond to measurements taken by secondary nodes located uniformly inside the circle with radius $d_{\operatorname{det}}\left(p_{1}\right)$ centered at $p_{1}$. We observe that although the difference between Cases $\mathrm{B}$ and $\mathrm{C}$ is negligible, both cases show some improvement $(\geq 50 \mathrm{~m}$ ) over Case A. The top three curves correspond to the worst-case scenario where the measurements are taken by secondary nodes located only at the intersection of the 


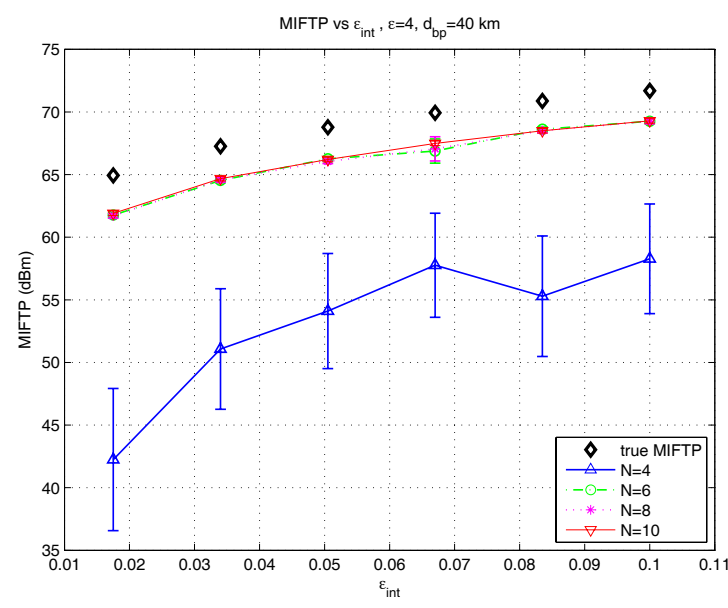

Fig. 7. MIFTP vs. maximum interference probability threshold, $\varepsilon_{\text {int }}$.

detection regions $d_{\operatorname{det}}\left(p_{1}\right)$ and $d_{\operatorname{det}}\left(p_{2}\right)$. A significant accuracy improvement is seen in Cases B and C ( $\geq 335 \mathrm{~m})$, more so in $\mathrm{B}$ than in $\mathrm{C}$, over Case A. The improvement for the worst-case scenario is much greater because the proposed compensation becomes more prominent when both transmitters contribute approximately equally to the measurements.

\section{B. MIFTP vs. distance}

Now consider the following configuration of cochannel transmitters: $\boldsymbol{\theta}_{p}=(0,0,80), \boldsymbol{\theta}_{a_{1}}=(20,20,40)$ and $\boldsymbol{\theta}_{a_{2}}=$ $(-20,20,40)$. We vary the position, $\left(0, y_{b}\right)[\mathrm{km}, \mathrm{km}]$, of the test node $b$, where $y_{b}$ ranges from 20 to 100 in increments of 10. The MIFTP of node $b$ is computed according to the approach presented in Section IV. The angle $\psi$ in (24) and (20) is discretized in increments of $\Delta \psi=\frac{\pi}{18}$ [rad]. Then the true MIFTP is computed according to (20). To approximate the MIFTP, $\mathcal{D}$ is computed for each trial. If $\mathcal{D} \neq \emptyset$, the MIFTP estimate is set to $-174 \mathrm{dBm}$ (which is the thermal noise floor at $1 \mathrm{~Hz}$ bandwidth at room temperature); otherwise, the estimated MIFTP is computed using (26). Note that computation of the true MIFTP requires the solution of $N_{T}=1+\frac{2 \pi}{\Delta \psi}$ one-dimensional optimization problems in (20), whereas the proposed approximation given in (26) requires only one.

In Fig. 3, we plot the true and estimated MIFTP as a function of the distance $d_{b p}$. As expected, the estimated MIFTP increases with distance, but is always smaller than the true MIFTP. Although the estimation is extremely conservative when $N=4$, a considerable improvement is seen when $N=6$. Using more measurements is only useful for distances smaller than $50 \mathrm{~km}$. On average, the estimated values are smaller than the true value by $5.35 \mathrm{~dB}$, when $N=6$ for all distances. We also plot the average interference probability (cf. (14)) perceived by victim nodes in $\bar{B}_{\text {cov }}(p)$ when node $b$ transmits at $\hat{s}_{b}^{*}(p)$ for $d_{b p}=20 \mathrm{~km}$ and $N=6$. As shown in Fig. 4, the interference probability surface is always less than the specified upper bound $\varepsilon_{\text {int }}=0.01$. The same has been observed for the values of $d_{b p}$ as well. Thus, the proposed MIFTP approximation can safely be used for opportunistic spatial spectrum access.

To study the effect of multiple primary transmitters, we consider the existence of another primary transmitter, $p^{\prime}$, in addition to $p$ on the same channel and time, with $\boldsymbol{\theta}_{p^{\prime}}=$ $(0,120,80)$. Since the two transmitters are very far apart $(120$ $\mathrm{km})$, we can ignore the effect of cochannel interference on localization. The true and approximate MIFTPs are calculated as $s_{b}^{*}=\min \left\{s_{b}^{*}(p), s_{b}^{*}\left(p^{\prime}\right)\right\}$ and $\hat{s}_{b}^{*}=\min \left\{\hat{s}_{b}^{*}(p), \hat{s}_{b}^{*}\left(p^{\prime}\right)\right\}$, respectively. Fig. 5 shows the variation of MIFTP as a function of distance. As node $b$ moves away from transmitter $p$ its MIFTP increases up to a certain level, then it decreases as it approaches transmitter $p^{\prime}$.

\section{MIFTP vs. shadowing noise and interference probability}

We set $d_{b p}=40 \mathrm{~km}$ and in Fig. 6 plot the estimated MIFTP as a function of the shadowing noise standard deviation, $\sigma_{W}$. As anticipated, the MIFTP decreases with increasing noise. This is because as the noise power increases, the localization error and the associated CRB increases, which in turn makes the MIFTP more conservative. For $N=4$ the estimated values are very loose, but they can be made reasonably tight using $N=6$ for any $\sigma_{W} \leq 9 \mathrm{~dB}$. Note that the decrease in true MIFTP is linear for all $\sigma_{W}$, but for the estimated MIFTP it is approximately linear only for $\sigma_{W} \leq 9$ $\mathrm{dB}$. For the extreme case of $\sigma_{W}>9 \mathrm{~dB}$, the estimated MIFTP is very loose and increasing $N$ helps very little. In Fig. 7 , we set $\sigma_{W}=8 \mathrm{~dB}$ and vary $\varepsilon_{\text {int }}$. We notice that as expected, the MIFTP becomes more conservative as the imposed interference constraint becomes tighter (lower $\varepsilon_{\text {int }}$ ). For $N \geq 6$, on average the estimated MIFTP is within $2.7 \mathrm{~dB}$ of the true value.

\section{CONCLUSION}

We presented a collaborative OSA scheme whereby multiple cochannel primary and secondary transmitters can co-exist in an interference-free condition. Based on a set of clustered measurements, secondary nodes estimate the power and location of primary transmitters that are located in their vicinity. Secondary nodes maintain a distributed database, called the T-map, containing location, power, and error estimates of cochannel nodes. The effect of cochannel interference is taken into account when global information about other cochannel transmitters becomes available via the T-map.

We developed a method for estimating the MIFTP available to a given secondary node in the presence of multiple cochannel primary transmitters. The proposed MIFTP estimation technique provides an approximate upper bound on the transmit power of a secondary transmitter. The construction, sharing, and updating of the T-map is performed collaboratively by secondary nodes throughout the network, making the scheme adaptive and robust. Our numerical results validate the accuracy of the proposed scheme when a sufficient number of signal strength measurements is available. The approximation for MIFTP is conservative, ensuring that secondary nodes do not cause harmful interference to the primary system.

In ongoing work, we are considering scenarios in which some or all of the nodes are equipped with directional antennas, which will impact estimation of the MIFTP. We are also developing a scheme that selects an "optimal" subset from a set of available measurements pertaining to a given 
cochannel transmitter, which will provide the most accurate location estimate. Such a measurement selection scheme would make collaborative sensing more robust to inaccurate signal measurement updates.

\section{REFERENCES}

[1] A. O. Nasif and B. L. Mark, "Collaborative opportunistic spectrum access in the presence of multiple transmitters," in Proc. IEEE Globecom'08, New Orleans, LA, Nov./Dec. 2008.

[2] S. Haykin, "Cognitive radio: brain-empowered wireless communications," IEEE J. Sel. Areas Commun., vol. 23, pp. 201-220, Feb. 2005.

[3] Q. Zhao and B. M. Sadler, "A survey of dynamic spectrum access," IEEE Signal Process. Mag., vol. 24, pp. 79-89, May 2007.

[4] C. Raman, R. D. Yates, and N. Mandayam, "Scheduling variable rate links via a spectrum server," in Proc. IEEE DySPAN'05, pp. 110-118, Nov. 2005.

[5] N. Devroye, P. Mitran, and V. Tarokh, "Achievable rates in cognitive radio," IEEE Trans. Inf. Theory, vol. 52, pp. 1813-1827, May 2006.

[6] R. Etkin, A. Parekh, and D. Tse, "Spectrum sharing for unlicensed bands," IEEE J. Sel. Areas Commun., vol. 25, pp. 517-528, Apr. 2007.

[7] S. Tang and B. L. Mark, "Modeling and analysis of opportunistic spectrum sharing with unreliable spectrum sensing," IEEE Trans. Wireless Commun., vol. 8, pp. 1934-1943, Apr. 2009.

[8] Y. Chen, Q. Zhao, and A. Swami, "Joint design and separation principle for opportunistic spectrum access in the presence of sensing errors," IEEE Trans. Inf. Theory, vol. 54, pp. 2053-2071, May 2008.

[9] A. Ghasemi and E. Sousa, "Collaborative spectrum sensing for opportunistic access in fading environments," in Proc. IEEE DySPAN'05, pp. 131-136, Nov. 2005.

[10] G. Ganesan and L. Ye, "Cooperative spectrum sensing in cognitive radio-part I: two user networks," IEEE Trans. Wireless Commun., vol. 6, pp. 2204-2213, June 2007.

[11] A. Sahai, N. Hoven, S. M. Mishra, and R. Tandra, "Fundamental tradeoffs in robust spectrum sensing for opportunistic frequency reuse," tech. rep., University of California, Berkeley, Mar. 2006.

[12] H. Celebi and H. Arslan, "Utilization of location information in cognitive wireless networks," IEEE Wireless Commun. Mag., vol. 14, pp. 6-13, Aug. 2007.

[13] H. Celebi and H. Arslan, "Cognitive positioning systems," IEEE Trans. Wireless Commun., vol. 6, pp. 4475-4483, Dec. 2007.

[14] S. Kim, H. Jeon, and J. Ma, "Robust localization with unknown transmission power for cognitive radio," in Proc. IEEE Milcom'07, Oct. 2007.

[15] J. K. Nelson, M. U. Hazen, and M. R. Gupta, "Global optimization for multiple transmitter localization," in Proc. IEEE Milcom'06, pp. 1-7, Oct. 2006.

[16] J. K. Nelson, M. U. Hazen, and M. R. Gupta, "An EM technique for multiple transmitter localization," in Proc. CISS'07, pp. 610-615, Mar. 2007.

[17] C. Raman, J. Kalyanam, I. Seskar, and N. Mandayam, "Distributed spatio-temporal spectrum sensing: an experimental study," in Proc. Asilomar Conf. Signals, Systems, Computers, (Pacific Grove, CA), pp. 2063-2067, Nov. 2007.

[18] R. Chen, J. M. Park, and J. H. Reed, "Defense against primary user emulation attacks on cognitive radio networks," IEEE J. Sel. Areas Commun., vol. 26, pp. 25-37, Jan. 2008.

[19] P. Mahonen, M. Petrova, and J. Riihijarvi, "Applications of topology information for cognitive radios and networks," in Proc. IEEE DySPAN'07, pp. 103-114, Apr. 2007.
[20] L.-C. Wang and A. Chen, "Effects of location awareness on concurrent transmissions for cognitive ad hoc networks overlaying infrastructurebased systems," IEEE Trans. Mobile Comput., vol. 8, pp. 577-589, May 2009.

[21] L. Qian, X. Li, J. Attia, and Z. Gajic, "Power control for cognitive radio ad hoc networks," in Proc. 15th IEEE Workshop LANMAN, pp. 7-12, June 2007.

[22] K. Hamdi, W. Zhang, and K. B. Letaief, "Power control in cognitive radio systems based on spectrum sensing side information," in Proc. IEEE ICC, pp. 5161-5165, June 2007.

[23] A. E. Leu, M. McHenry, and B. L. Mark, "Modeling and analysis of interference in Listen-Before-Talk spectrum access schemes," Int. $J$. Network Mgmt, vol. 16, pp. 131-147, Mar./Apr. 2006.

[24] B. L. Mark and A. O. Nasif, "Estimation of interference-free power for opportunistic spectrum access," IEEE Trans. Wireless Commun., vol. 8, pp. 2505-2513, May 2009.

[25] B. L. Mark and A. E. Leu, "Local averaging for fast handoffs in cellular networks," IEEE Trans. Wireless Commun., vol. 6, pp. 866-874, Mar. 2007.

[26] N. C. Beaulieu, A. A. Abu-Dayya, and P. J. MacLane, "Estimating the distribution of a sum of independent lognormal random variables," IEEE Trans. Commun., vol. 43, pp. 2869-2873, Dec. 1995.

[27] L. L. Scharf, Statistical Signal Processing: Detection, Estimation, and Time Series Analysis. New York: Addison-Wesley, 1991.

[28] A. O. Nasif and B. L. Mark, "Opportunistic spectrum access with multiple transmitters," Tech. Rep. TR-GMU-NAPL-Y09-N1, George Mason University, Feb. 2009. Available at http://napl.gmu.edu.

[29] A. O. Nasif and B. L. Mark, "Measurement clustering criteria for localization of multiple transmitters," in Proc. Conf. Info. Sciences Systems (CISS'09), Baltimore, MD, Mar. 2009.

[30] J. Rissanen, "Modeling by shortest data description," Automatica, vol. 14, pp. 465-471, 1978.

[31] S. Verdú, Multiuser Detection. New York: Cambridge University Press, 1998.

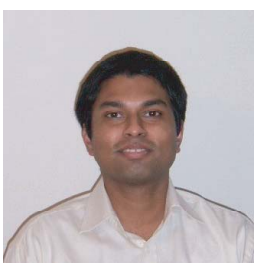

Ahmed O. Nasif (S'05) received the B.S.E.E. degree from Bangladesh University of Engineering and Technology, Dhaka, Bangladesh, in 2003, the M.S.E. degree in Electrical Engineering from Wright State University, Dayton, $\mathrm{OH}$, in 2005, and the $\mathrm{Ph} . \mathrm{D}$. in Electrical and Computer Engineering at George Mason University in 2009. Currently, he is a postdoctoral research fellow at George Mason University. His research interests lie in wireless communications, signal processing, information theory, and estimation theory.

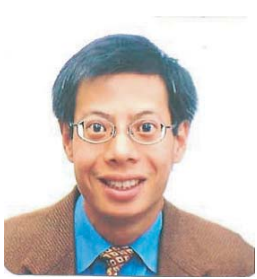

Brian L. Mark (M'91-SM'08) received the B.A.Sc. degree in Computer Engineering with an option in Mathematics from the University of Waterloo, Canada, in 1991 and the Ph.D. in Electrical Engineering from Princeton University, Princeton, NJ, in 1995. He is an Associate Professor in the Dept. of Electrical and Computer Engineering at George Mason University. His research interests lie in the design, modeling, and analysis of communication systems and networks. 